\title{
Design of Power Communication Data Network Management System and Implementation of Data Acquisition Module
}

\author{
Yue Zhang' ${ }^{1}$, Zefeng Jian' ${ }^{1}$, Hao Wang ${ }^{2}$ \\ ${ }^{1}$ Information and Communication Branch, Inner Mongolia Power (GROUP) CO., LTD, Hohhot 010000, Inner Mongolia \\ Autonomous Region, China \\ 2Inner Mongolia Coal Construction Engineering (Group) Corporation, Hohhot 010000, Inner Mongolia Autonomous \\ Region, China
}

\begin{abstract}
The power communication network is a separate network from the power grid whose primary purpose is to ensure the power grid's safe operation. This paper expounds the composition of the comprehensive network management architecture of the power communication data network and the implementation of the data acquisition module in the network management system through theoretical analysis, for the reference of relevant personnel, in order to better promote the collection of power grid communication network data.
\end{abstract}

Keywords: Electric power communication; Network management system design; Data acquisition

Publication date: November 2021; Online publication: November 30, 2021

\section{Introduction}

Power communication network plays an important role in the operation of power grid and is also an important part of the national power grid economy. Power communication data network management covers a wide range of business. It is necessary to continuously strengthen the management of power communication data network management system in order to better promote the operation of power communication data network management system. Therefore, the design of data module in power communication data network management system is of great significance for the development of power communication data network management system ${ }^{[1]}$. According to the requirements of business, function and data of power communication data network management system, it is imperative to improve the data module. The data module can realize the alarm monitoring of power data and the fault treatment of power data. Through the data module, the flow analysis and configuration audit of power grid communication data can be realized, and the real-time inspection and maintenance of power grid communication can ensure the safe operation of power grid communication network; The data module can also collect dynamic resource data, collect alarm data and sort out logs, reflect the data situation of the current power communication network through the analysis of performance data ${ }^{[2]}$, and ensure the safe implementation and development of power communication services. In addition, it can also meet the functional records of power communication data network management, and ensure the safe operation of power grid communication data network management system.

\section{Integrated network management architecture of power communication data network}

At present, the integrated network management architecture of power communication data network mainly includes acquisition adaptation layer, application layer, information representation layer and network 
element layer. The specific architecture is shown in Figure 1.

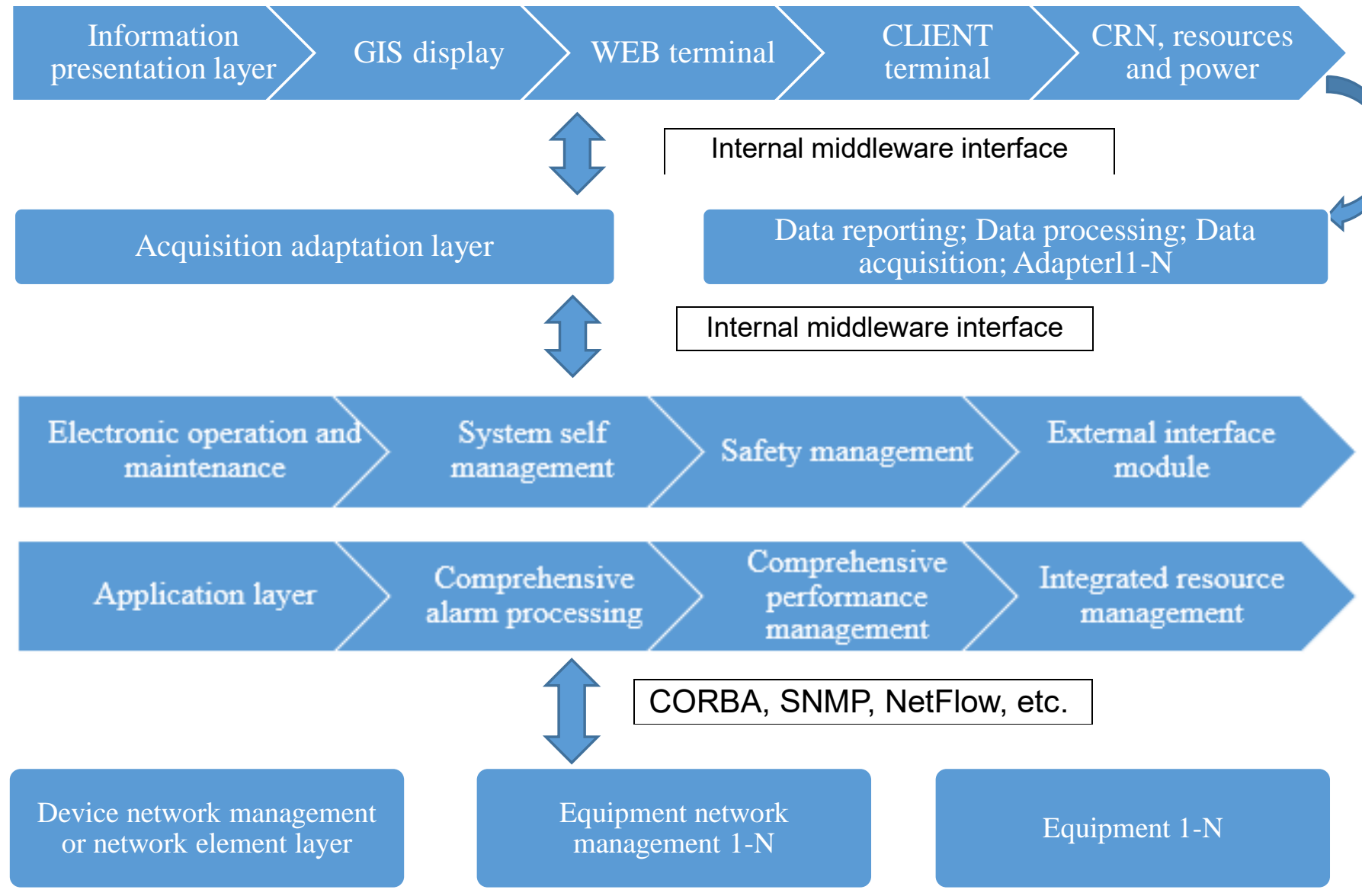

Figure 1. Design framework of power communication data network management system

(1) Device network management or network element layer: The required access interfaces of managed devices and unmanaged devices are different. The access interfaces of unmanaged devices are provided by the network element layer, which can be accessed by accessing the interfaces provided by the network element layer, so as to obtain the data information required by the device; The access interface of the managed equipment is provided by the equipment network management, including the private interface, which can access the data information, so as to realize the data sharing and real-time information exchange of all access equipment.

(2) Acquisition adaptation layer: Among the current popular interface protocols, Common Object Request Broker Architecture (CORBA), Simple Network Management Protocol (SNMP) and NetFlow are widely used. At present, the interface protocols adopted by the equipment connected to the power communication network are different. Therefore, in order to avoid inaccessibility, an acquisition adapter layer needs to be set in the power communication network management to connect the acquisition adapter, in this way, each type of network management equipment can be accessed. Different adapters correspond to different network management systems and different network element types.

(3) Application layer: The main function of this level is to realize the interaction of functions, including the real-time sharing of data information, data information processing, data information analysis of each network element interface and north interface. In this level, it can realize the warning processing of data information and connect each functional module in the power communication network management system. It is the main information interaction layer. In order to better realize 
the role transformation, through the special interface function, realize the interaction of power communication network management system resources and support various electronic equipment, so that power communication network management system can operate stable.

(4) Information presentation layer: The main function of this layer is to display relevant information to users, realize the visualization of various data information, and facilitate users to intuitively understand the contents of various data information. The superior docking layer of the information presentation layer is the application layer and only connects with the application layer. The content of information data is displayed to the client in browser mode and client server mode.

\section{Realization of data acquisition function of power communication data network management system}

\subsection{Processing of alarm data}

Configuration and alarm data constitute the data type collected, in which the configuration data can provide the required data to the resource management subsystem, so as to realize network management; Alarm is an abnormal prompt message sent by power communication network equipment during operation, which is sent to operation and maintenance personnel through work order or short message to accurately judge the fault location, type and severity, and carry out targeted maintenance. When implementing alarm data processing, the number of network equipment alarms shall be considered to make the system design of data network management highly scalable and flexible, meet the correct technical selection, and make the interface interactive and maintainable ${ }^{[3]}$.

\subsection{Collection of performance data}

The realization of module functions includes initialization operation, data acquisition, remote control and etc. In order to effectively realize performance data acquisition, it is necessary to pay attention to remote control and do a good job in each initial operation, which requires the assistance of predefined interface protocol, and then carry out accurate operation through the required adaptation function. In addition, the designed task table needs to be imported to allow remote instructions to control subsequent overload operations, and then change the configuration accurately and quickly to meet the operation needs. In addition, when collecting various data, there must be a background description to effectively collect performance related data, and then obtain extremely accurate new performance data through scientific incremental analysis and carry out detailed operations.

\subsection{Collection and control of configuration data}

The collection and control of configuration data shall first realize the remote function, establish the basic configuration and operation and maintenance debugging command library of various stations, and complete the configuration and commissioning of new stations through command call. After a series of initialization operations, carry out daily monitoring and data collection for field equipment and services at all levels designated in the background ${ }^{[4]}$. During operation, the acquisition and control functions shall be realized through the transmission function interface according to the specific session protocol.

\subsection{Realization of acquisition control function}

Data acquisition and control are based on alarm information and combined with acquisition business logic for exception management and storage operation. The collection of alarm data covers the remote control link. It is also necessary to carry out corresponding data collection and complete the scientific call of instructions required by the predefined interface. The acquisition control will carry out subsequent operations through the start-up instructions in combination with the actual situation. In the specific 
acquisition process, it shall be carried out according to the specific subnet protocol. In the process of operation, the differences of subnet acquisition interfaces should be considered, so as to call the function interface in advance and realize the function to a great extent ${ }^{[5]}$.

\subsection{Protocol adaptation execution}

The implementation of protocol adaptation needs the assistance of control framework to scientifically and comprehensively analyze the acquisition instructions. After the session is established, the obtained information should be converted accordingly, for example, specify a format, and then provide an interface that satisfies its return requirements. Generally speaking, the operation of alarm collection can be triggered through the collection control framework, then deeply analyze the manufacturer's protocol, complete the protocol adaptation operation, and then realize it through the callback interface. Due to the system can receive alarm information in various environments to a great extent, it can bring practical and effective guarantee to the whole operating system through scientific and automatic processing. If the system cannot operate anymore, for example, it is in a resting state, we can use destroy callback method to quickly clear all the original information of the interface. For NetFlow protocol, it does not need polling, so it is easy to handle ${ }^{[6]}$. After the main program is started, NetFlow protocol can be automatically collected. At the same time, the program will collect the received data and input it into the database, convert it into alarm data, transmit it to the upper layer and process it. SNMP trap will send trap packets without polling. It can accurately analyze the alarm information and complete the write operation of the database. For SNMP protocol, the SNMP interface in the acquisition module will perform SNMP get operation, and the agent will quickly package the extracted effective data, convert it into external format, then perform the encapsulation operation, and send it to the manager in the form of SNMP package to realize the implementation of protocol adaptation.

\section{Summary}

To sum up, the current power communication gateway system mainly includes four technical support layers, namely, acquisition adaptation layer, application layer, information representation layer and network element layer. Through the elaboration and analysis of the architecture, the implementation of data acquisition module is proposed. And through the collection of alarm data, performance data and configuration data, further realize the acquisition control function and protocol adaptation execution function, so as to complete the realization of the data acquisition module of power communication data network.

\section{Disclosure statement}

The author declares no conflict of interest.

\section{References}

[1] Zhang JW, Wang WL, Lan B, et al., 2021, Research on Health Status Data Checking Scheme of Power Communication Optical Cable Network. Adhesion, 46(06): 103-105 + 115.

[2] Tong XZ, 2021, Effective Integration Strategy Power Information and Power Communication Technology. Informatization Construction, 2021(06): 62.

[3] Wang YM, 2021, Design and Research of Encryption Scheme for Power Automatic Wireless Communication. Housing and Real Estate, 2021(05): 124-125.

[4] Zheng YT, Zhang F, Huang LS, et al., 2021, Research and Application of Data Transmission Method 
for Power Safety Zone 1. Scientific and Technological Innovation, 2021(13): 59-60.

[5] Feng RJ, Li XD, Yang SF, Zeng XY, 2021, Application of MD5 Algorithm in Power Communication. Practical Electronics, 2021(09):52-53 + 47.

[6] Xing WT, Wang XY, Zhao YH, 2021, Research on Communication Scheduling and Duty Automation Technology of Power System. Journal of Shandong Electric Power College, 24(02): 58-61. 